\title{
Deducing Ink Spreading Curves from Reflection Spectra Acquired Within Printed Color Images
}

\author{
R. D. Hersch`, M. Brichon, T. Bugnon and M. Hebert \\ School of Computer and Communication Sciences, Ecole Polytechnique Fédérale de Lausanne (EPFL), \\ Switzerland \\ E-mail: rd.hersch@epfl.ch
}

\begin{abstract}
The Yule-Nielsen modified spectral Neugebauer model (YNSN) enhanced for accounting for ink spreading in the different ink superposition conditions provides accurate spectral predictions, but requires one to measure the reflectances of special halftone calibration patches in order to compute the ink spreading curves mapping nominal ink surface coverage to effective ink surface coverage. Printing special halftone calibration patches within the borders of the print pages is cumbersome, since these special patches need to be cut out before assembling the final printed product. In the present contribution, we calibrate the ink spreading curves directly from the printed images by fitting them so as to minimize a distance metric between predicted reflectances and measured reflectances at selected relatively uniform locations. We compare the prediction accuracy of the so-calibrated YNSN model with the prediction accuracy of the same model calibrated by the spectral reflectances of classical uniform calibration patches. Interestingly, when the model is calibrated from image tiles originating from the same or from a similar color image as the one comprising the test tiles, better prediction results are obtained than when performing a classical calibration on $50 \%$ halftone patches printed in all superposition conditions. (- 2009 Society for Imaging Science and Technology. [DOI: 10.2352/J.ImagingSci.Technol.2009.53.3.030502]
\end{abstract}

\section{INTRODUCTION}

The goal of a color reproduction system is to be able to reproduce input colors as accurately as possible. This is not a trivial task since the human visual system is very sensitive to small color differences. In printing systems, there are many factors influencing the range of printable colors: the inks, the substrate (paper, plastic, glass, etc.), the illumination conditions, and the halftones. Spectral reflection prediction models are helpful in studying the influence of these factors and in creating printer characterization profiles for the purpose of color management. ${ }^{1}$

One of the fundamental aspects that a spectral reflection prediction model has to consider is how the inks spread on the paper, a phenomenon also referred to as physical or mechanical dot-gain. With an ink spreading model accounting for physical dot-gain, a spectral reflection prediction model is able to predict reflectance spectra in function of ink surface coverages for three or four inks. ${ }^{2-4}$ In order to provide accurate predictions, such an ink spreading model must

\section{IS\&T Member.}

Received Oct. 11, 2008; accepted for publication Feb. 11, 2009; published online Apr. 23, 2009.

$1062-3701 / 2009 / 53(3) / 030502 / 7 / \$ 20.00$. take into account not only the interaction between an ink halftone and paper, but also the interaction between an ink halftone and the other superposed inks. One proposed solution is to use multiple ink spreading curves, also called tone reproduction curves, to characterize the physical dotgain of the ink halftones on paper and in all solid ink superposition conditions. ${ }^{5}$ For CMYK halftone prints, such an approach requires the characterization of 20 ink spreading curves. ${ }^{6}$

Ink spreading is computed by taking into account the respective physical dot-gains of one ink halftone (e.g., at $50 \%$ nominal surface coverage) printed in different superposition conditions, i.e., alone on paper, in superposition with one ink, in superposition with two inks, and in superposition with three inks. Effective dot surface coverages are fitted separately for each superposition condition by minimizing a difference metric between measured reflection spectrum and predicted reflection spectrum. This yields, for each superposition condition, an ink spreading curve mapping nominal to effective surface coverages. ${ }^{5}$ For the spectral prediction of a color halftone, nominal surface coverage values are converted into effective coverage values by weighting the contributions of the different ink spreading curves according to the ratios of the halftone's colorant surfaces.

Printing special uniform halftone calibration patches on separate pages or within the borders of the print pages is cumbersome, because these special patches need to be removed before assembling the final printed product. However, in present color printing systems, such special calibration patches are necessary for measuring both the dot-gains of halftones and the densities of the solid inks. In the present contribution, we perform a first step toward the elimination of special halftone patches by calibrating the ink spreading curves on color tiles selected from printed color images.

Within the color images to be reproduced, whose nominal cyan, magenta, yellow, and black (cmyk) surface coverage values are known from prepress, we first select color tiles as uniform as possible, distributed across the gamut of the image colors. In a second step, we calibrate simultaneously all ink spreading curves of halftones printed with cyan, magenta, and yellow (cmy) inks by fitting their effective surface coverages at $50 \%$ nominal surface coverage so as to minimize a metric expressing the difference between predicted reflectances and measured reflectances of the selected image 
tiles. In a third step, we calibrate the ink spreading curves of the black halftones with reflection spectra from calibration tiles incorporating a minimal amount of black ink.

After calibration of the ink spreading curves, we use the ink spreading enhanced spectral prediction model to predict the effective surface coverages (a) of relatively uniform test tiles located within the considered images and (b) of 125 cyan, magenta, and yellow, respectively, 625 cyan, magenta, yellow, and black halftone patches well distributed within the cmy, respectively, cmyk color space (Prediction Accuracy section). We quantify the accuracy of spectral halftone patch predictions by converting measured and predicted spectra first to CIE-XYZ and then to CIELAB. The distance defined by the CIELAB $\Delta E_{94}$ color difference formula gives a measure of the visually perceived distance between measured and predicted spectra.

\section{THE INK SPREADING ENHANCED YULE-NIELSEN SPECTRAL NEUGEBAUER MODEL (EYNSN)}

Many different phenomena influence the reflection spectrum of a color halftone patch printed on a diffusely reflecting substrate (e.g., paper). These phenomena comprise the surface (Fresnel) reflection at the interface between the air and the paper, light scattering and reflection within the substrate (i.e., the paper bulk), and the internal (Fresnel) reflections at the interface between the paper and the air.

The lateral scattering of light within the paper substrate and the internal reflections at the interface between the paper and the air are responsible for what is generally called optical dot-gain, also known as the Yule-Nielsen effect. In addition, due to the printing process, deposited ink dot surface coverages are generally larger than nominal coverages, yielding the physical dot-gain. Such effective ink dot surface coverages depend on the inks, the paper, and also the specific ink superposition conditions; i.e., the superposition of ink halftones and solid inks.

One of the first color prediction models is the Neugebauer model. ${ }^{7}$ In its original form, it predicts the CIE$\mathrm{XYZ}$ tristimulus values of a color halftone patch as the sum of the tristimulus values of their individual colorants weighted by their fractional area coverages $a_{i}$. By considering the reflection spectra $R_{i}$ of colorants instead of their respective tristimulus values, we obtain the spectral Neugebauer equations. ${ }^{8}$ They predict the reflection spectrum of a printed color halftone patch as a function of the reflection spectra of its individual colorants (also called Neugebauer primaries):

$$
R(\lambda)=\sum_{i} a_{i} R_{i}(\lambda)
$$

With $k$ inks, there are $2^{k}$ colorants: white, the $k$ single ink colorants and all the combinations of superpositions of solid inks. For example, the red colorant is the superposition of the magenta and yellow inks. When the ink layers are printed independently one from another, the individual colorant area coverages can be deduced from the ink surface coverages by the Demichel equations. ${ }^{9}$ These equations are

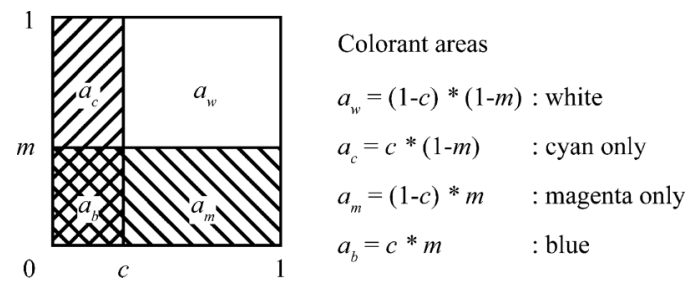

Figure 1. Deducing according to the Demichel equations the colorant surface coverages of white $\left(a_{m}\right)$, cyan only $\left(a_{c}\right)$, magenta only $\left(a_{m}\right)$, and blue $\left(a_{b}\right)$ from the ink surface coverages of the cyan $(c)$ and magenta $(m)$ ink dots.

shown in Figure 1 for the case of two inks, but can be easily extended to three or four inks. ${ }^{3}$

Since the Neugebauer model neither takes explicitly into account the lateral propagation of light within the paper bulk nor the internal reflections (Fresnel reflections) at the paper-air interface, its predictions are not accurate. Yule and Nielsen ${ }^{10}$ modeled the nonlinear relationship between the reflectances of a color halftone, the reflectances of its colorants and their surface coverages by a power function whose exponent $n$ can be optimized according to the reflectance of a limited set of color halftone patches. Viggiano ${ }^{11}$ applied the Yule-Nielsen relationship to the spectral Neugebauer equations, yielding the Yule-Nielsen modified spectral Neugebauer model (YNSN):

$$
R(\lambda)=\left(\sum_{i} a_{i} R_{i}(\lambda)^{1 / n}\right)^{n}
$$

The YNSN model has been used by many researchers for the characterization of printing systems. ${ }^{2,3,12-14}$ It therefore plays a significant role in color management systems. Recently, we extended the Yule-Nielsen modified spectral Neugebauer model with an ink spreading model (EYNSN). ${ }^{5}$ The ink spreading model is used to account for the physical dot-gain in the different ink dot superposition conditions; i.e., the fact that an ink dot spreads out differently when printed alone on the paper, or when printed in superposition with other ink dots. The resulting effective ink dot surface coverages are usually larger than the intended nominal ink dot surface coverages.

Spectral predictions using the EYNSN model are performed according to Figure 2. First, the effective single ink halftone surface coverages for the contributing superposition conditions are determined from nominal surface coverages by using the ink spreading curves $f_{i / j k}$ established during model calibration. There is one ink spreading curve for each ink halftone in each superposition condition; i.e., an ink halftone superposed with paper only, and superposed with one, two, or three given solid inks. The effective surface coverages of the ink dots forming a color halftone are obtained by a weighted average of the previously established ink spreading curves. From the effective ink dot surface coverages, we compute the effective colorant surface coverage with the Demichel equations. Finally, from these effective colorant coverages, the YNSN model computes the predicted reflection spectrum. 


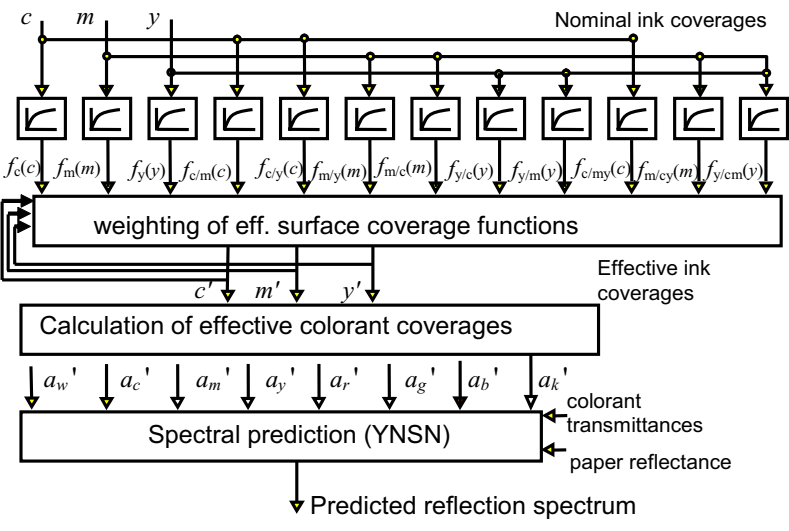

Figure 2. The ink spreading enhanced Yule-Nielsen modified spectral Neugebauer model for three inks with nominal ink surface coverages $c$, $m$, and $y$, effective single ink halftone dot surface coverages $f_{i / i k}$ of ink $i$ superposed with solid inks $j$ and $k$, effective ink surface coverages $c^{\prime}, m^{\prime}$, and $y^{\prime}$, and effective colorant surface coverages $a_{w}^{\prime} a_{c}^{\prime}$ to $a_{k}^{\prime}$.

Table I. List of the ink spreading curve indicia.

\begin{tabular}{lllll}
\hline Cyan & Magenta & Yellow & \multicolumn{2}{c}{ Black } \\
\hline$c$ & $m$ & $y$ & $k$ & $k / y$ \\
$c / m$ & $m / c$ & $y / c$ & $k / c$ & $k / c y$ \\
$c / y$ & $m / y$ & $y / m$ & $k / m$ & $k / m y$ \\
$c / m y$ & $m / c y$ & $y / c m$ & $k / c m$ & $k / c m y$ \\
\hline
\end{tabular}

\section{THE INK SPREADING EQUATIONS}

The proposed ink spreading model considers the ink spreading behavior (physical dot-gain) of each ink in each different ink superposition condition. The amount of ink spreading depends on whether the ink halftone is printed alone on paper or in superposition with one or more other inks.

An ink spreading curve maps the nominal surface coverages of an ink into its effective surface coverages; i.e., to the surface that the ink effectively covers once printed on paper or superposed with other solid inks. There is one ink spreading curve for each ink halftone in each superposition condition. For example, a cyan halftone may be printed (1) alone, (2) superposed with solid magenta, (3) with solid yellow, (4) with solid black, (5) with solid magenta and solid yellow, (6) with solid magenta and solid black, (7) with solid yellow and solid black, and (8) with solid magenta, yellow, and black. There are eight different ink spreading curves for each ink, yielding a total of 32 ink spreading curves. However, since any halftone superposed with solid black yields solid black, ink spreading curves where one ink halftone is superposed with solid black are discarded. ${ }^{6}$ The remaining ink spreading curves are listed in Table I.

Note that the ink spreading curves used for calibrating the spectral prediction model are defined for superposition conditions composed of solid inks only. There is, for example, an ink spreading curve for a cyan halftone over solid magenta, but no ink spreading curve for a cyan halftone over $50 \%$ magenta.
The following notation is used for specifying the ink spreading curves: ink halftone/superposed inks. For example, $f_{c / m}$ refers to the cyan halftone over solid magenta ink spreading curve, $f_{c / m y}$ refers to the cyan over solid magenta and solid yellow ink spreading curve, and $f_{c}$ refers to the ink spreading curve of a cyan halftone printed alone. An ink spreading curve characterizes how a given ink halftone behaves in a given superposition condition, when printing that halftone at surface coverages varying between $0 \%$ and $100 \%$.

With the "classical" calibration relying on specially printed calibration patches, ${ }^{5}$ each ink spreading curve is calibrated by fitting effective surface coverages minimizing the sum of square differences between measured spectral reflectance and predicted spectral reflectance of an ink halftone patch (e.g., the halftone at 50\% nominal surface coverage). The spectral measurements span the visible wavelength range $(380-730 \mathrm{~nm})$, and in order to avoid the ambiguity between chromatic black and pure black ${ }^{15}$ are extended to cover the near infrared (NIR) wavelength range (730-850 nm). The NIR wavelength range enables distinguishing the light absorbing pigment-based black ink and the $c m y$ inks which are dye-based and do not absorb light in the NIR wavelength range.

In order to obtain the effective surface coverages of the inks forming a color halftone, the effective single ink halftone surface coverages deduced from the ink spreading curves are weighted according to the surface coverages of the colorants contributing to that color halftone. In the case of the three cyan magenta and yellow inks, since there are four possible superposition conditions for each ink halftone, the effective surface coverage of an ink is the weighted average of four different ink spreading curves. The relative weights are given by the surface coverages of the colorants superposed with the considered ink halftone. For example, for a patch printed with surface coverages of 0.4 cyan, 0.3 magenta, and 0.2 yellow, the superposition dependent colorant surface coverages weighting the ink spreading curves of cyan are $(1-0.3) \times(1-0.2)=0.56$ for unprinted paper, $0.3 \times(1-0.2)=0.24$ for the magenta colorant, $(1-0.3) \times 0.2=0.14$ for the yellow colorant, and $0.3 \times 0.2=0.06$ for the red colorant (superposition of magenta and yellow).

Equations (3) weight the ink spreading curves of each ink halftone according to the corresponding effective surface coverages of the underlying colorants formed by the other inks. We solve these equations iteratively, starting by assigning first the nominal ink halftone coverages to the effective ink halftone coverages. ${ }^{6}$ Four to five iterations ensure sufficient convergence to determine the effective ink halftone surface coverages.

$$
\begin{aligned}
c^{\prime}= & \left(1-m^{\prime}\right)\left(1-y^{\prime}\right) f_{c}(c)+m^{\prime}\left(1-y^{\prime}\right) f_{c / m}(c) \\
& +\left(1-m^{\prime}\right) y^{\prime} f_{c / y}(c)+m^{\prime} y^{\prime} f_{c / m y}(c), \\
m^{\prime}= & \left(1-c^{\prime}\right)\left(1-y^{\prime}\right) f_{m}(m)+c^{\prime}\left(1-y^{\prime}\right) f_{m / c}(m) \\
& +\left(1-c^{\prime}\right) y^{\prime} f_{m / y}(m)+c^{\prime} y^{\prime} f_{m / c y}(m),
\end{aligned}
$$




$$
\begin{gathered}
y^{\prime}=\left(1-c^{\prime}\right)\left(1-m^{\prime}\right) f_{y}(y)+c^{\prime}\left(1-m^{\prime}\right) f_{y / c}(y) \\
+\left(1-c^{\prime}\right) m^{\prime} f_{y / m}(y)+c^{\prime} m^{\prime} f_{y / c m}(y), \\
k^{\prime}=\left(1-c^{\prime}\right)\left(1-m^{\prime}\right)\left(1-y^{\prime}\right) f_{k}(k)+c^{\prime}\left(1-m^{\prime}\right)\left(1-y^{\prime}\right) f_{k / c}(k) \\
+\left(1-c^{\prime}\right) m^{\prime}\left(1-y^{\prime}\right) f_{k / m}(k)+c^{\prime} m^{\prime}\left(1-y^{\prime}\right) f_{k / c m}(k) \\
+\left(1-c^{\prime}\right)\left(1-m^{\prime}\right) y^{\prime} f_{k / y}(k)+c^{\prime}\left(1-m^{\prime}\right) y^{\prime} f_{k / c y}(k) \\
+\left(1-c^{\prime}\right) m^{\prime} y^{\prime} f_{k / m y}(k)+c^{\prime} m^{\prime} y^{\prime} f_{k / c m y}(k) .
\end{gathered}
$$

Since a cyan, magenta, or yellow halftone over solid black is assumed to yield black, in Eq. (3), the superposition conditions corresponding to an ink halftone over solid black have been discarded. We have therefore four superposition conditions for the cyan, magenta, and yellow ink halftones and eight superposition conditions for the black ink halftone.

\section{SELECTING UNIFORM COLOR AREAS WITHIN COLOR IMAGES FOR CALIBRATION PURPOSES}

In a previous work, ${ }^{16}$ we simplified the calibration process by avoiding the necessity of performing spectral reflectance measurements on the calibration patches. We deduced ink spreading curves from scanned RGB images of the calibration patches. In the present work, we perform the calibration of the ink spreading curves by acquiring spectra located within printed color images that have been characterized as reference images. Avoiding special calibration patches simplifies the print workflow. This is especially true for large print presses, where printing special patches on the border of the printed pages requires for their subsequent elimination sophisticated in-line cutting equipment.

The first step consists in selecting within the color images present on the printed page color areas as uniform as possible. For this purpose, the corresponding prepress multilevel color images are downsampled to $150 \mathrm{dpi}$ and converted from display RGB to CIELAB (e.g., by assuming that the display is an sRGB display). Then, each color image is scanned horizontally and vertically, by advancing in $2 \mathrm{~mm}$ intervals, and by indexing and analyzing the $5 \times 5 \mathrm{~mm}^{2} \mathrm{im}-$ age tiles at the corresponding image locations. A nonuniformity criterion is associated with each color image tile.

As nonuniformity criterion, we rely on the root mean square (rms) of the standard deviation of the CIELAB pixel values located within the considered image tile:

$$
s=\sqrt{\left(\sigma\left(L^{\star}\right)^{2}+\sigma\left(a^{\star}\right)^{2}+\sigma\left(b^{\star}\right)^{2}\right) / 3} .
$$

The larger this rms standard deviation, the larger the nonuniformity of the corresponding tile.

All image tiles are indexed from their smallest to their largest nonuniformity criterion value. In order to form the calibration set, we scan the set of tiles, from the most uniform to the least uniform tile and successively select the 50 tiles with the smallest nonuniformity values which, with the exception stated below, are at a distance of at least $\Delta E_{a b}=6$ from all previously selected calibration set patches. In order to provide a small amount of redundancy, we allow, within the select set, subsets of maximal two tiles having between them a $\Delta E_{a b}$ distance of $2<\Delta E_{a b}<6$.

In order to evaluate the accuracy of the present approach, we also create from the indexed image tiles a test set comprising 30 patches. Members of the test set are also as uniform as possible but must differ from the members of the calibration set and be apart by at least $\Delta E_{a b}=6$ one from another.

The selection of the calibration and test patch set is performed within the color fruits or textile images (Supplement A, available on the IS\&T website, www.imaging.org). Tiles from the same image are used for deducing the ink spreading curves both in the three ink cmy spectral prediction model and in the four ink cmyk spectral prediction model. Supplement A shows the two color images and the location of the calibration (blue rectangles) and test (green rectangles) image tile. Supplement B (available on the IS\&T website, www.imaging.org) shows the corresponding calibration and test patches, together with their respective rms standard deviation expressed in CIELAB.

\section{CALIBRATION OF THE INK SPREADING CURVES WITH SPECTRA MEASURED ON THE SELECTED IMAGE TILES}

Experiments were carried out on a Canon Pixma Pro 9500 printer, with color images printed on Canon MP-101 matte paper with classical clustered dot halftoning, at a resolution of $600 \mathrm{dpi}$ and a screen frequency of $100 \mathrm{lpi}$. The color images are halftoned with classical mutually rotated screens (Adobe Photoshop ${ }^{\mathrm{TM}}$ ). The black ink is a pigment based ink absorbing in the near infrared wavelength range (cartridge PGI-9MBK). Reflectance spectra were measured with a Datacolor MF-45 spectrophotometer (geometry: $45^{\circ} \mathrm{d}: 0^{\circ}$ ) at $10 \mathrm{~nm}$ intervals in the range between $380 \mathrm{~nm}$ and $850 \mathrm{~nm}$. The reflectance spectra of paper and of the solid colorants (all combinations of solid ink superpositions) have been measured. The $n$-value of the extended Yule-Nielsen spectral Neugebauer model used in connection with these ink-jet prints is 8 . The present calibration aims at computing the ink spreading curves in order to calibrate the model as accurately as possible, without printing specially conceived halftone patches. For cyan, magenta, and yellow inks only, according to Eq. (3), we have 12 ink spreading curves. We associate with each ink spreading curve mapping nominal to effective surface coverages in a given superposition condition a dot-gain curve, with the dot-gain being defined as the effective surface coverage minus the nominal surface coverage.

Since there is no dot-gain at zero and at $100 \%$ ink halftone surface coverage, we approximate each dot-gain curve by a parabola starting at point $(0,0)$, going through point $\left(0.5, d g_{i / j k}(0.5)\right)$ and ending at point $(1,0)$, with $d g_{i j j k}(0.5)=f_{i / j k}(0.5)-0.5$.

We fit unknown effective surface coverages $f_{i / j k}(0.5)$ at $50 \%$ nominal surface coverage yielding the corresponding dot-gains $d g_{i / j k}(0.5)$ by minimizing a distance function be- 


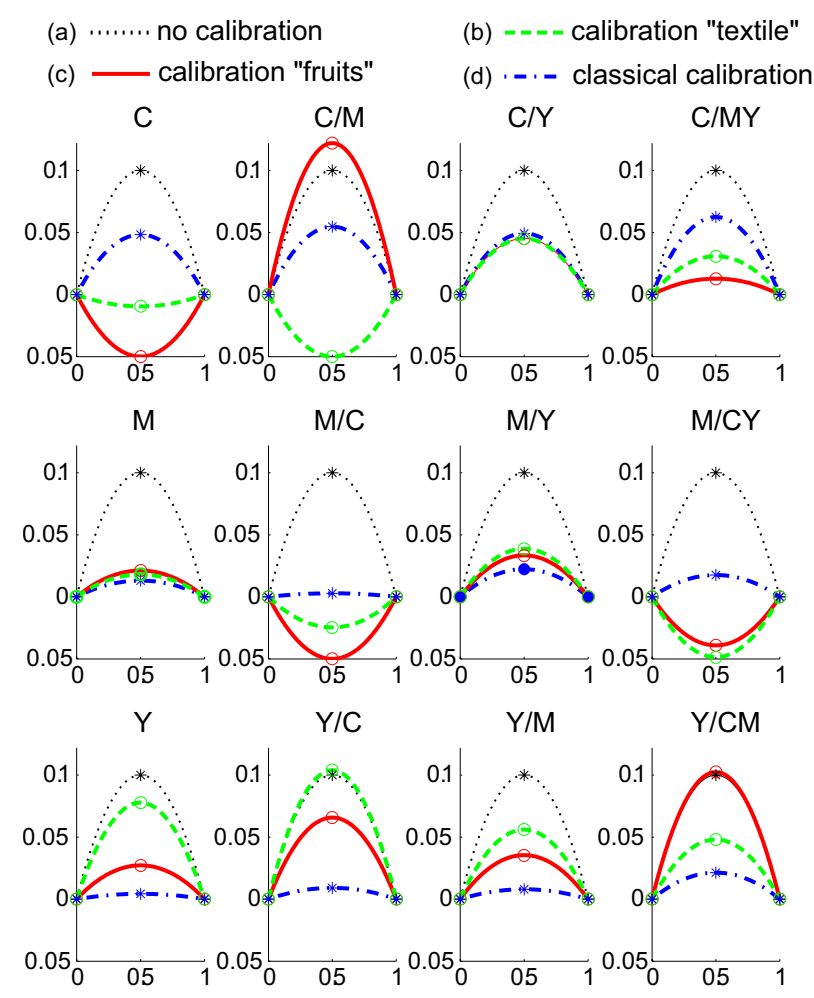

Figure 3. The dot-gain curves in the different superposition conditions, for cmy prints, (a) without calibration, preset at 10\% dot-gain, (b) fitted from the reflectance of nearly uniform color areas located in color image textile, (c) fitted from the reflectance of nearly uniform color areas located in color image fruits, and (d) fitted from specially printed halftone patches at $50 \%$ nominal surface coverage (classical calibration).

tween predicted and measured reflection spectra at the selected color image calibration tile locations. As distance function $E$, we sum up over all selected calibration tiles the sum of squared differences between measured and predicted density spectra components, with the relation between reflectance $R(\lambda)$ and density spectrum $D(\lambda)$ given by $D(\lambda)=-\log _{10} R(\lambda)$. For the three cmy inks, we simultaneously fit the effective surface coverages values $f_{c}(0.5)$, $f_{c / m}(0.5), f_{c / y}(0.5), f_{c / m y}(0.5), f_{m}(0.5), f_{m / y}(0.5), f_{m / c}(0.5)$, $\left.f_{m / c y}(0.5), \quad f_{y}(0.5), \quad f_{y / c}(0.5), \quad f_{y / m}(0.5), \quad f_{y / c m}(0.5)\right)$ by minimizing

$$
E=\sum_{\substack{\text { calibration } \\ \text { tiles }}} \sum_{\lambda}\left(D_{i, \lambda, \text { meas }}-D_{i, \lambda, \text { predicted }}\right)^{2} .
$$

The fitting procedure uses matlab's constrained minimization fmincon function, with an initial dot gain of $10 \%$ at $50 \%$ nominal surface coverages, a lowest dot-gain limit at -0.05 and a highest dot-gain limit at +0.15 .

Figure 3 shows the dot-gain curves obtained with the present calibration procedure using the calibration tiles either from the fruits image or from the textile image, compared with the dot-gain curves obtained with the "classical" calibration on specially printed halftone patches.

The dot-gain curves fitted from the textile image tiles (green dashed lines) behave in most cases similarly to the dot-gain curves fitted from the fruit image tiles (red continuous lines). However, the dot-gain curves fitted from the textile or fruit image tiles are considerably different from those (blue dashed-dotted lines) obtained by the classical calibration, i.e., by printing the $50 \%$ halftone calibration patches alone on paper or on top of other solid inks. The calibration patches selected within the color images are not representative of the full color gamut. Therefore, optimizing the dot-gain curves for patches representative of a given image yields different dot-gain curves than the ones optimized over colors located at or near the gamut boundaries formed by the classical calibration set of $50 \%$ ink halftones, printed alone or in superposition with one or more solid inks. The dot-gain curves deduced by fitting from image tiles do not necessarily represent a "real" physical dot-gain, but rather a "compensation" bringing the model predictions closer to the spectral measurements.

In the case of $c m y k$ prints, we divide the set of calibration tiles into two subsets, one having less and the second having equal or more than $20 \%$ surface coverage of the black ink. The first subset of tiles is used for simultaneously fitting the effective surface coverage values according to the $12 \mathrm{~cm} y$ superposition conditions and to deduce the corresponding ink spreading curves. The second subset of tiles is used to fit the effective surface coverages of black halftones at $50 \%$ nominal surface coverage in all the eight black halftone superposition conditions and deduce the corresponding ink spreading curves $f_{k}, f_{k / c}, f_{k / m}, f_{k / y}, f_{k / c m}, f_{k / c y}, f_{k / m y}, f_{k / c m y}$.

For patches printed with the four cyan, magenta, yellow, and black inks, Figure 4 shows the deduced dot-gain curves for the cyan, magenta, and yellow halftones in all superposition conditions as well as the additional dot-gain curves of the black ink on top of paper and of other inks, obtained with the present calibration procedure, compared with the dot-gain curves obtained with the classical calibration. For the cyan and magenta ink halftones, the dot-gain curves deduced from the fruits image tiles printed with cmyk inks (red continuous lines) are similar to ones deduced from corresponding image tiles printed with $c m y$ inks (green dashed lines). For the yellow ink halftones, this similarity is less pronounced, probably due to the fact that the yellow ink diffuses part of the incident light. As in the case of three inks, substantial differences appear when comparing the dotgain curves deduced from tiles of the fruits image and those deduced from a classical cmyk calibration (blue dasheddotted lines). ${ }^{6}$

\section{PREDICTION ACCURACY}

Let us first describe the prediction accuracies for the three ink cmy prints. We compare the spectral prediction accuracies of the ink spreading enhanced YNSN model (a) calibrated according to the classical calibration and (b) calibrated with spectral measurements of image tiles located within the color images. As a "worst case," we also consider the prediction accuracies obtained without calibration; i.e., dot-gain curves relying on a physical dot-gain of 0.1 at a nominal halftone surface coverage of 0.5 . 
(a) $\cdots . .$. no calibration

(c) - - classical calibration

(b) calibration "fruits" 4 inks

(d) =- =- calibration "fruits" 3 inks
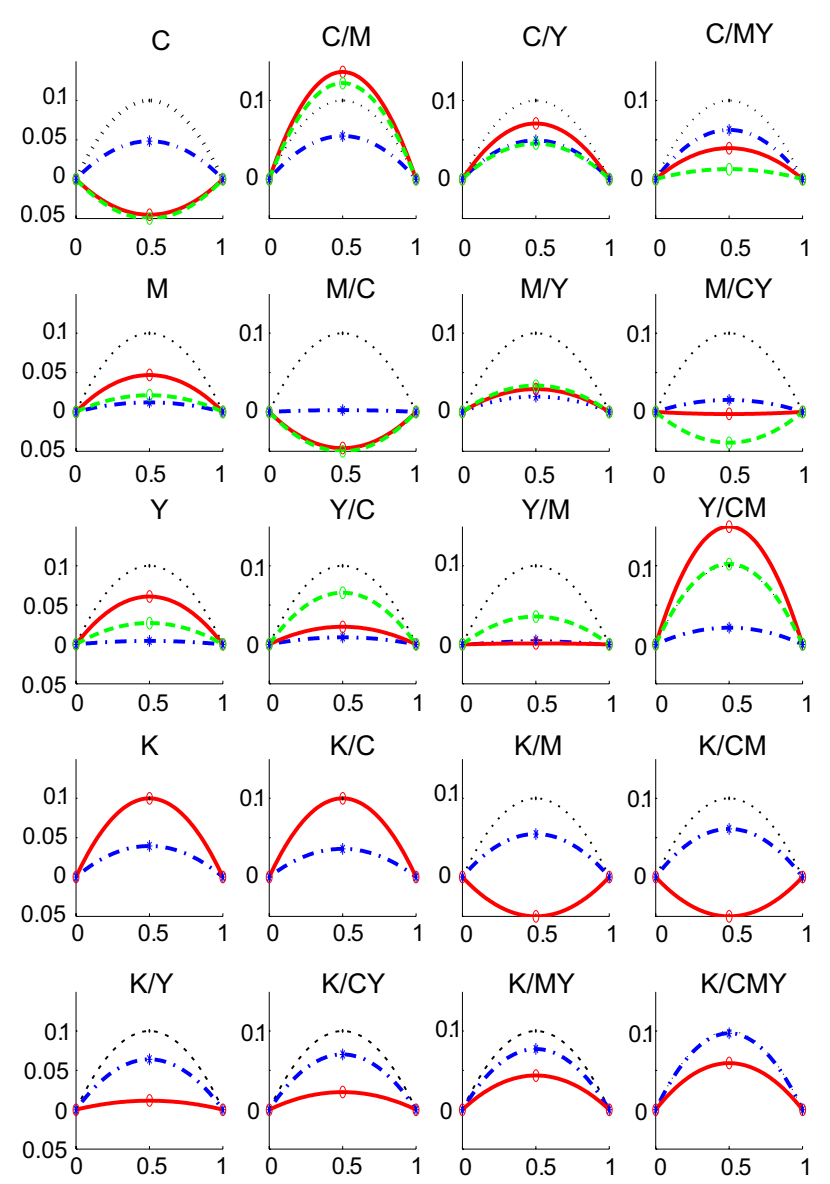

Figure 4. The dot-gain curves in the different superposition conditions, for cmyk prints, (a) set at 10\% dot-gain, (b) fitted from the reflectance of nearly uniform color areas located in cmyk color image fruits, (c) fitted from specially printed halftone patches at 50\% nominal surface coverage (classical cmyk calibration), and (d) for comparison purposes, the cmy dot-gain curves fitted from the reflectance of nearly uniform color areas located within the three ink cmy fruits image.

We have several test sets: one test set comprising 30 color tiles within the fruit image, a second test set with 30 tiles within the textile image, a third test set comprising specially created $125 \mathrm{~cm} y$ halftone patches printed at all combinations of nominal surface coverages $0,0.25,0.5,0.75$, and 1 , and a last cmyk test set comprising patches printed at all combinations of surface coverages $0,0.25,0.5,0.75$, and 1 . The same fruits and textile images are printed both with cmy inks and with cmyk inks, with an undercolor removal performed in a standard manner by the Photoshop software package. For cmy prints, Table II gives the prediction accuracies of the ink spreading enhanced Yule-Nielsen spectral prediction model, for no calibration, for the classical calibration, and for a calibration with image tiles extracted from the textile or from the fruit images present on the printed page.

Unsurprisingly, the highest prediction accuracy is obtained with calibration tiles originating from the same image as the test tiles. For the test sets of both images, when a classical calibration is performed with specially printed $50 \%$
Table II. In the case of cmy prints, comparison of the prediction accuracies for the considered test sets, when calibrating the ink spreading curves with the classical calibration, or with tiles extracted from color images.

\begin{tabular}{lcll} 
& $\begin{array}{l}\text { Mean } \\
\Delta E_{94}\end{array}$ & $\begin{array}{l}95 \% \\
\text { quantile } \\
\Delta E_{94}\end{array}$ & $\begin{array}{l}\text { Max } \\
\Delta E_{94}\end{array}$ \\
\hline Test set Textile & & & \\
No calibration & 3.33 & 5.10 & 5.29 \\
Classical calibration & 1.86 & 3.18 & 3.44 \\
Calibration set textile & 0.92 & 1.91 & 2.05 \\
Calibration set fruits & 1.10 & 2.49 & 2.60 \\
\hline Test set Fruits & & & \\
No calibration & 3.16 & 4.49 & 4.92 \\
Classical calibration & 1.49 & 2.55 & 2.69 \\
Calibration set textile & 1.13 & 2.70 & 2.89 \\
Calibration set fruits & 0.92 & 1.96 & 2.26 \\
\hline Test set 125 patches & & & \\
No calibration & 2.51 & 4.31 & 4.48 \\
Classical calibration & 1.00 & 2.62 & 3.21 \\
Calibration set fextile & 1.67 & 4.38 & 4.95 \\
Calibration set fruits & 1.50 & 4.05 & 5.91 \\
\hline
\end{tabular}

halftone patches, the average prediction accuracy is significantly lower than with a calibration relying on image tiles. However, the classical calibration yields strongly improved prediction results on the test set of 125 uniformly distributed cmy patches. This may be explained by the fact that the classical calibration patches with ink halftones at 50\% superposed with solid inks are "closer" to the patches where we have all possible combinations of inks at $0 \%, 25 \%, 50 \%$, $75 \%$, and $100 \%$ surface coverages. On the other side, calibrating the spectral prediction models with tiles originating from the color images is more representative for predicting other tiles from the same or from a similar color image.

Table III gives the prediction accuracies of the ink spreading enhanced YNSN model with tiles from the same image locations as in Table II, but printed with cmyk inks, by performing under color removal. As an additional test set, we consider 625 halftones with all possible combinations of cmyk inks at $0 \%, 25 \%, 50 \%, 75 \%$, and $100 \%$ surface coverages.

Here also, using the calibration tiles from the same image as the test set yields the best prediction results. With test tiles located within the color images, the classical calibration yields slightly worse prediction results than with the calibration with tiles from the same or from another similar image. However, in contrast to the three ink cmy prediction model, on the 125 or 625 set of patches well distributed across the printer gamut, the classical calibration does not necessarily yield better prediction accuracies than the calibration from image tiles. This phenomenon deserves a more detailed analysis. 
Table III. For cmyk printed calibration and test tiles, comparison of prediction accuracies as a function of the different calibration procedures.

\begin{tabular}{llll}
\hline & & $95 \%$ & \\
& Mean & $\begin{array}{l}\text { quantile } \\
\Delta E_{94}\end{array}$ & $\begin{array}{c}\text { Max } \\
\Delta E_{94}\end{array}$ \\
\hline Test set Textile & & & \\
No calibration & 3.07 & 4.62 & 5.87 \\
Classical calibration & 1.48 & 2.47 & 2.91 \\
Calibration set textile & 1.06 & 2.02 & 2.17 \\
Calibration set fruits & 1.19 & 2.73 & 3.30 \\
\hline Test set Fruits & & & \\
No calibration & 2.68 & 4.95 & 5.11 \\
Classical calibration & 1.60 & 2.79 & 3.27 \\
Calibration set fextile & 1.46 & 3.00 & 3.29 \\
Calibration set fruits & 1.15 & 1.83 & 3.77 \\
\hline Test set 125 patches, & & & \\
with UCR & & & \\
No calibration & 3.55 & 5.71 & 6.74 \\
Classical calibration & 1.70 & 3.23 & 3.73 \\
Calibration set fextile & 1.62 & 3.31 & 5.03 \\
Calibration set fruits & 1.37 & 2.50 & 3.31 \\
\hline Test set 625 patches & & & \\
No calibration & 2.85 & 5.20 & 6.33 \\
Classical calibration & 1.29 & 2.43 & 3.29 \\
Calibration set fextile & 1.32 & 3.25 & 5.71 \\
Calibration set fruits & 1.22 & 3.00 & 6.59 \\
\hline
\end{tabular}

\section{CONCLUSIONS}

Printing systems capable of recharacterizing and recalibrating themselves are a major objective for tomorrow's color printing workflows. ${ }^{17}$ We perform a first step toward that objective by deducing ink spreading curves in all superposition conditions from locations present within the printed color images. These ink spreading curves are, besides the spectra of paper of the printed solid ink superpositions, essential elements for calibrating the ink spreading enhanced Yule-Nielsen modified spectral Neugebauer model.

Ink spreading curves deduced from image tiles whose color pixels vary according to a standard deviation of less than $\sigma_{\mathrm{Lab}}=5$ in CIELAB space provide an excellent calibration of the model enabling accurately predicting the reflectance spectra of other tiles from the same or from a similar image having a standard deviation similar to the one of the calibration tiles. An open question is to what extent nonuniform image tiles would also allow to fit the ink spreading curves.
Since an individual image does not represent in a uniform manner the colors located within the printable gamut, dot-gain curves deduced from tiles located within such an image may be biased. For example, within a given color image, there may be only one image tile containing a sufficient surface coverage of given ink. The ink spreading curves of that ink will therefore be strongly biased by that image tile. Future research will have to deal with the problem of establishing confidence criteria specifying how representative a given ink spreading curve extracted from color image tiles is with respect to the corresponding printing process.

\section{ACKNOWLEDGMENTS}

The authors would like to thank M. Herbert Janser, Wifag Maschinenfabrik, Bern, for having pointed to the necessity of simplifying as much as possible the calibration of the spectral prediction model. The authors also thank the Swiss National Science Foundation for their funding effort, Grant No. 200020-105119.

\section{REFERENCES}

${ }^{1}$ R. Bala, "Device characterization", in Digital Color Imaging Handbook, edited by G. Sharma (CRC Press, Boca Raton, FL, 2003) pp. 269-379.

${ }^{2}$ R. Balasubramanian, "Optimization of the spectral Neugebauer model for printer characterization”, J. Electron. Imaging 8, 156-166 (1999).

${ }^{3}$ D. R. Wyble and R. S. Berns, "A critical review of spectral models applied to binary color printing”, Color Res. Appl. 25, 4-19 (2000).

${ }^{4} \mathrm{~T}$. Ogasahara, "Verification of the predicting model and characteristics of dye-based ink jet printer", J. Imaging Sci. Technol. 48, 130-137 (2004).

${ }^{5}$ R. D. Hersch and F. Crété, "Improving the Yule-Nielsen modified spectral Neugebauer model by dot surface coverages depending on the ink superposition conditions”, Proc. SPIE 5667, 434-445 (2005).

${ }^{6}$ Th. Bugnon, M. Brichon, and R. D. Hersch, "Simplified ink spreading equations for CMYK halftone prints”, Proc. SPIE 6807, 680717 (2008).

${ }^{7}$ H. E. J. Neugebauer, "Die theoretischen Grundlagen des Mehrfarbendrucks", Z. Wiss. Phot. 36, 36-73 (1937), reprinted in Proc. SPIE 1184, 194-202 (1989). English translation: "The theoretical basis of multicolor letterpress printing”, Color Res. Appl. 30, 322-331 (2005).

${ }^{8}$ J. A. S. Viggiano, "The color of halftone tints", Proc. TAGA, 37, 647-661 (1985).

${ }^{9}$ M. E. Demichel, Procédé 26, 17-21 (1924).

${ }^{10}$ J. A. C. Yule and W. J. Neilsen, "The penetration of light into paper and its effect on halftone reproductions", Proc. TAGA 3, 65-76 (1951).

${ }^{11}$ J. A. S. Viggiano, "Modeling the color of multi-colored halftones", Proc. TAGA 42, 44-62 (1990).

${ }^{12} \mathrm{~K}$. Iino and R. S. Berns, "Building color management modules using linear optimization I. Desktop”, J. Imaging Sci. Technol. 42(1), 79-94 (1998).

${ }^{13}$ A. U. Agar and J. P. Allebach, "An iterative cellular YNSN method for color printer calibration”, Proc. IS\& T/SID 6th Color Imaging Conference (IS\&T, Springfield, VA, 1998) pp. 197-200.

${ }^{14} \mathrm{~S}$. Zuffi and R. Schettini, "An innovative method for spectral-based printer characterization”, Proc. SPIE 4663, 1-7 (2002).

${ }^{15}$ T. Bugnon, M. Brichon, and R. D. Hersch, "Model-based deduction of CMYK surface coverages from visible and infrared spectral measurements of halftone prints", Proc. SPIE 6493, 649310 (2007).

${ }^{16}$ N. Garg, A. K. Singla, and R. D. Hersch, "Calibrating the Yule-Nielsen modified spectral Neugebauer model with ink spreading curves derived from digitized RGB calibration patch images", J. Imaging Sci. Technol. 52, 40908 (2008).

${ }^{17}$ R. Bala, G. Sharma, V. Monga, and J.-P. Van de Capelle, "Twodimensional transforms for device color correction and calibration", IEEE Trans. Image Process. 14, 1172-1186 (2005). 